\title{
Probing the Local Order of Single Phospholipid Membranes Using Grazing Incidence X-Ray Diffraction
}

\author{
C. E. Miller, ${ }^{1}$ J. Majewski, ${ }^{1}$ E. B. Watkins, ${ }^{1,2}$ D. J. Mulder, ${ }^{3}$ T. Gog, ${ }^{4}$ and T. L. Kuhl ${ }^{3,5}$ \\ ${ }^{1}$ Manuel Lujan Neutron Scattering Center, Los Alamos National Laboratory, Los Alamos, New Mexico 87545, USA \\ ${ }^{2}$ Biophysics Graduate Group, University of California, Davis, 95616, USA \\ ${ }^{3}$ Department of Chemical Engineering and Materials Science, University of California, Davis, 95616, USA \\ ${ }^{4}$ Advanced Photon Source, Argonne National Laboratory, Argonne, Illinois 60439, USA \\ ${ }^{5}$ Department of Biomedical Engineering, University of California, Davis, 95616, USA
}

(Received 26 June 2007; published 6 February 2008)

\begin{abstract}
We report the first grazing incidence $\mathrm{x}$-ray diffraction measurements of a single phospholipid bilayer at the solid-liquid interface. Our grazing incidence x-ray diffraction and reflectivity measurements reveal that the lateral ordering in a supported DPPE (1, 2-Dipalmitoyl-sn-Glycero-3-Phosphoethanolamine) bilayer is significantly less than that of an equivalent monolayer at the air-liquid interface. Our findings also indicate that the leaflets of the bilayer are uncoupled in contrast to the scattering from free standing phosphatidylcholine bilayers. The methodology presented can be readily implemented to study more complicated biomembranes and their interaction with proteins.
\end{abstract}

DOI: 10.1103/PhysRevLett.100.058103

PACS numbers: 87.16.D-, 61.05.cm, 82.45.Mp

One of the most intriguing issues in membrane biology today is their lateral organization [1]. Do lipids and proteins mix uniformly within cellular membranes or are they arranged in discrete domains? For example, the spacing, size, and transmembrane asymmetry of cellular membrane domains may be key properties that govern function [2]. If these domains or "rafts" are laterally ordered, grazing incidence x-ray diffraction (GIXD) has the potential to address the following questions: (i) What are the packing properties of lipids including repeat distances and molecular tilts in the ordered portion of the membrane? (ii) How big are the effective sizes of scattering domains? (iii) Is there coupling between the two phospholipid leaflets or do they scatter independently? Salditt and co-workers have demonstrated the power of using GIXD to characterize multilayer stacks of lipids and proteins in humid environments [3,4]. Here, the study of single biomembranes in bulk water is demonstrated which has potential for more biologically relevant conditions and simplification of structure. In addition, specular X-ray reflectivity (XR) measurements are sensitive to the electron density distribution along the surface normal, complementing the precise inplane packing properties and correlation length scales of the ordered (diffracting) portions of the film provided by GIXD [5,6]. By combining such measurements with knowledge of the chemical structure of the molecules, the organization of thin films can be extracted in a quite general approach. At conventional photon energies of $\sim 10 \mathrm{keV}, \mathrm{x}$ rays are strongly attenuated and incoherently scattered by water, limiting XR and GIXD studies to thin films at the solid-air or liquid-air interface. Recently, some straightforward modifications such as increasing the x-ray energy and minimizing the path of $\mathrm{x}$ rays through water have overcome these limitations and enabled the study of single membranes in bulk water [5-8].
In this study, XR and GIXD were utilized to determine the structure of a single, supported bilayer of DPPE (1,2Dipalmitoyl-sn-Glycero-3-Phosphoethanolamine) at the single crystal quartz-water interface. The inner and outer leaflet of the DPPE bilayer were deposited by the Langmuir-Blodgett and Langmuir-Schaffer (L-B/S) dipping techniques [9]. Both depositions were done at a surface pressure of $45 \mathrm{mN} / \mathrm{m}$ and $23^{\circ} \mathrm{C}$, which corresponds to the gel-phase of DPPE. Details of the solid-liquid interface cell were given previously [5]. Synchrotron x-ray measurements on DPPE bilayers were carried out at beam lines 9-ID and 6-ID at the advanced photon source (APS), Argonne National Laboratory (ANL) (Argonne, IL) at wavelengths of 0.690 and $0.545 \AA$. By modeling the measured specular XR, detailed information on the average electron density distribution in the direction normal to the interface was determined [10].

For the GIXD experiments, Bragg peaks arise from scattered intensity along the scattering vector component parallel to the quartz substrate, $q_{x y}\left(\right.$ or $\left.q_{\|}\right) \cong(4 \pi / \lambda) \times$ $\sin \left(2 \theta_{x y} / 2\right)$, where $2 \theta_{x y}$ is the angle between the incident and diffracted beam projected onto the quartz plane [11]. The width of the Bragg peaks, corrected for the instrumental resolution [12], gives the finite size of the crystalline domains in the direction of the reciprocal scattering vector $q_{x y}$ (the 2D in-plane crystalline coherence length, $L_{x y}$ ) according to the Scherrer formula [13]. In-plane and outof-plane resolutions for the bilayer experiments were $\Delta q_{x y}=0.0267 \AA^{-1}$ and $\Delta q_{z}=0.001 \AA^{-1}$. The GIXD images were recorded using FujiFilm BAS-5000 image plates (IP) and a Mar345 image plate detector with 2-4 s exposure times. Comparison studies on DPPE monolayers were carried out using the liquid surface diffractometer (with $\Delta q_{x y}=0.008 \AA^{-1}$ ) at the BW1 (undulator) beam 
line at HASYLAB, DESY (Hamburg, Germany). The theory of XR and GIXD has been presented in detail elsewhere $[14,15]$.

First, the formation of a single homogenous DPPE bilayer at the solid-liquid interface was verified by reflectivity measurements [5]. Reflectivity profiles of a single bilayer in contrast to a single monolayer leaflet at the water-air interface (at $45 \mathrm{mN} / \mathrm{m}$ ) are shown in Fig. 1(a). The corresponding electron density profiles from box model fitting procedures [10] are shown in Fig. 1(b). The structure of the outer bilayer leaflet matches well to the monolayer at $45 \mathrm{mN} / \mathrm{m}$, with similar headgroup and tail region densities and thicknesses. A thin water layer between the inner DPPE leaflet and the quartz substrate was
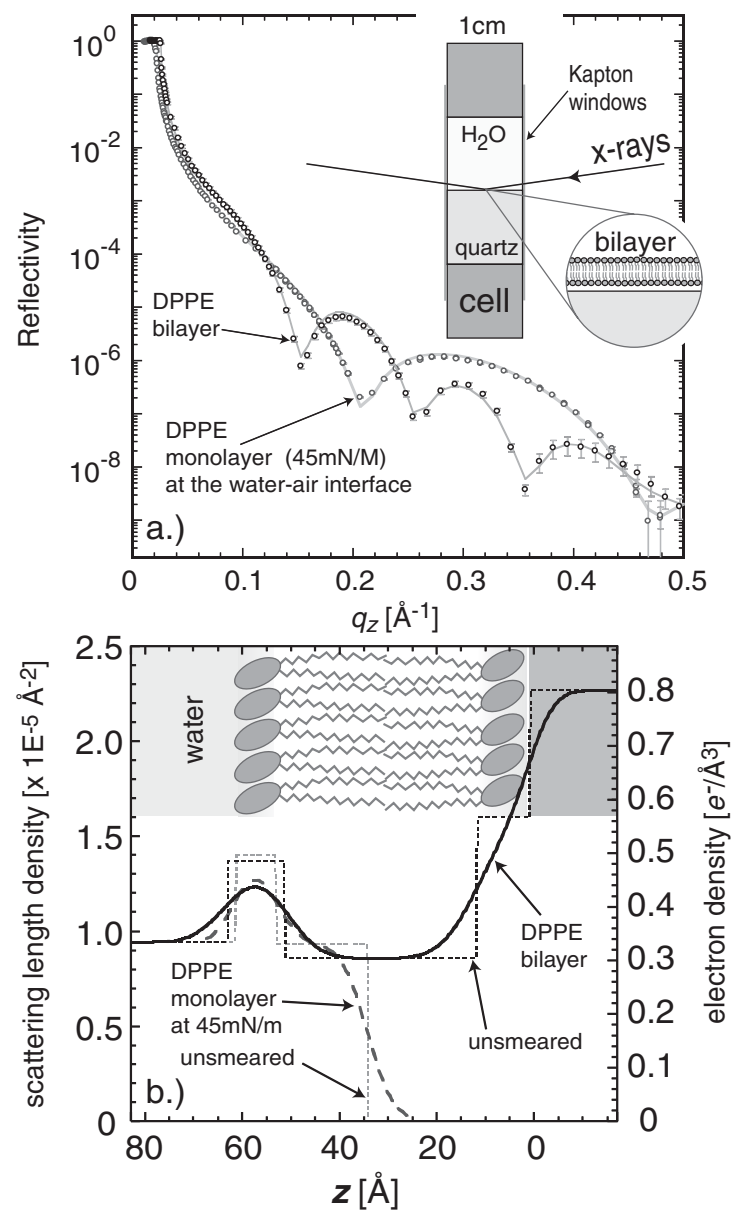

FIG. 1. (a) Reflectivity profile for a DPPE bilayer deposited at $45 \mathrm{mN} / \mathrm{m}$ at the quartz- $\mathrm{H}_{2} \mathrm{O}$ interface and a DPPE monolayer at the $\mathrm{H}_{2} \mathrm{O}$-air interface $(45 \mathrm{mN} / \mathrm{m})$. The vertical momentum transfer vector $q_{z}$ is $4 \pi \sin \theta / \lambda$, where $\theta$ is the angle of incidence to the interface and $\lambda$ is the wavelength of $\mathrm{x}$-ray beam. Symbols represent measured data and solid lines are the fits corresponding to the scattering length density profiles in (b). (Inset) Schematic of the experimental cell. (b) Scattering length density and electron density profiles corresponding to the fits in (a) and obtained by box model fitting procedures. The electron density profile for the DPPE monolayer has been oriented for comparison with the outer leaflet of the bilayer. not required to model the reflectivity data but the presence of such a layer cannot be ruled out. Comparable studies with phosphatidylcholine (PC) lipids, which are known to be more hydrated than PE lipids [16], require a $4-5 \AA$ water layer [5].

For GIXD experiments, the incident x-ray beam was adjusted to strike the surface at the vertical momentum transfer vector $q_{z}=0.62 q_{c}$, where $q_{c}=0.0256 \AA^{-1}$ is the critical scattering vector for total external reflection for quartz submerged in water $(e$-densities of quartz and water are $0.797 e^{-} / \AA^{3}$ and $0.334 e^{-} / \AA^{3}$, respectively). At this angle the incident wave is totally reflected, while the refracted wave becomes evanescent traveling along the interface.

Figure 2 shows the IP for Bragg reflection from a DPPE bilayer in bulk water. Bragg peaks are the intensity resolved in the $q_{x y}$ direction and integrated over the $q_{z}$ range $\left(-0.04 \AA^{-1}<q_{z}<0.3 \AA^{-1}\right)$. Bragg rods are the intensity resolved in the $q_{z}$ direction and integrated over the $q_{x y}$ range $\left(1.46 \AA^{-1}<q_{x y}<1.54 \AA^{-1}\right)$ of the Bragg peak. The magnitudes of momentum transfer vectors were calculated using the instrument geometry and x-ray wavelength and confirmed by powder diffraction from standard salts. For the DPPE bilayer, Bragg scattering can be observed at $q_{x y}=1.5 \AA^{-1}$ (Fig. 3) and extends perpendicularly from the interface up to $q_{z} \approx 0.25 \AA^{-1}$.

For all DPPE bilayers studied (a dozen measurements across 3 sample preparations), only one in-plane Bragg peak from the lipid tails was observed suggesting a hexagonal lattice with a hexagonal unit cell dimension of $a_{H}=4.84 \pm 0.01 \AA$. Because of the limited $q_{x y}$ resolution

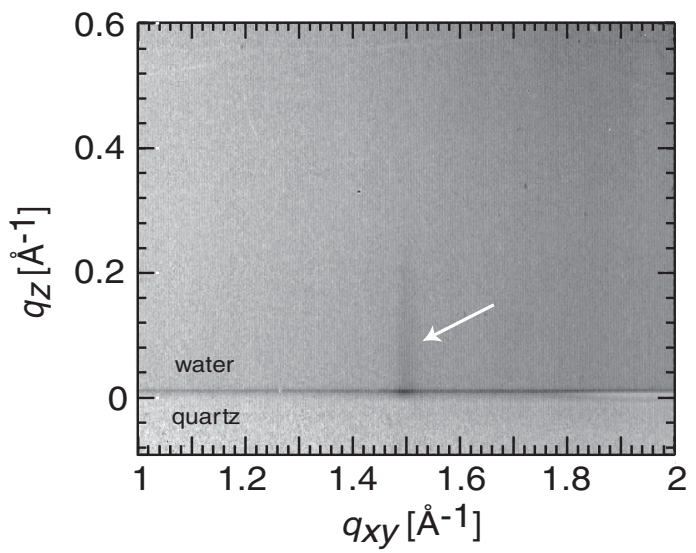

FIG. 2. Diffracted intensity distribution from a single DPPE bilayer membrane on a quartz substrate deposited by L-B/S deposition at $45 \mathrm{mN} / \mathrm{m}$. To enhance the visibility of the diffraction peak the image was produced by subtracting the intensity distribution of a bare quartz substrate in contact with water. The projection of the quartz substrate is defined as $q_{z}=0 \AA^{-1}$. The scattered intensity along the line slightly above $q_{z}=0 \AA^{-1}$ is the Vineyard-Yoneda peak [22]. The Bragg rod at $q_{x y}=1.5 \AA^{-1}$ extends from the quartz interface up to $q_{z} \approx 0.25 \AA^{-1}$ and is due to the lateral ordering of the alkyl chains of DPPE. 
it was not possible to discriminate between a single, broad peak due to a hexagonal unit cell and a slightly distorted hexagonal or oblique unit cell where the splitting of the 3 Bragg peaks in $q_{x y}$ was not resolvable. Assuming a hexagonal unit cell, the $d$ spacing between lipid alkyl tails in a DPPE bilayer membrane is slightly larger than their spacing in a monolayer at $45 \mathrm{mN} / \mathrm{m}$ before depositing on a substrate. These values correspond to an area per molecule (of the diffracting portion of the bilayer) of $40.57 \AA^{2}$ as compared to $40.06 \AA^{2}$ for the monolayer at $45 \mathrm{mN} / \mathrm{m}$. The observed $d$ spacing is comparable to a DPPE monolayer between $30 \mathrm{mN} / \mathrm{m}$ and $45 \mathrm{mN} / \mathrm{m}$, and indicates that the packing efficiency decreases with deposition on the support [17]. Additionally, the lateral distance over which the lipid tails are in registry with one another in the bilayer $(194 \pm 50 \AA)$ is significantly less than that of the monolayer at $45 \mathrm{mN} / \mathrm{m}(570 \pm 10 \AA)$ or even at $20 \mathrm{mN} / \mathrm{m}$ (400 $\pm 10 \AA$ ). Table I provides a summary of the GIXD analysis. These precise structural measurements establish that the resulting bilayer is considerably less ordered than the original monolayer yet retains solid phase domains that are resolvable by GIXD measurements.

In Bragg rod analysis, the intensity of the DPPE bilayer (Fig. 4) was concentrated close to the interface $\left(q_{z}=0\right)$ similar to a monolayer at $45 \mathrm{mN} / \mathrm{m}$. Cylindrical objects were used to approximate the scattering units of the alkyl tails [18]. Parameters corresponding to the cylinder's height $\left(L_{c}\right)$, radius, tilt angle from the surface normal, direction of tilt within unit cell, and root mean-square molecular displacement (Debye-Waller factor) normal to the interface were adjusted using a Levenberg-Marquardt least-squares fitting algorithm to obtain the best possible fit.

A key objective of the Bragg rod analysis was to determine whether the lipid leaflets of the bilayer scatter inde- pendently or as one coupled entity. The DPPE bilayer rod (Fig. 4) clearly shows a similar FWHM as in the monolayer case at $45 \mathrm{mN} / \mathrm{m}$ suggesting a similar $L_{c} \approx 21 \AA$. Because the maximum intensity along the Bragg rod of the bilayer was concentrated near the quartz interface, it was possible to model the data with a family of fits with various lengths and tilts. To limit the number of possible models we restricted the length of the scattering cylinder $\left(L_{c}\right)$ to that of alkyl tails of a monolayer $(\sim 21 \AA)$ and bilayer ( $\sim 22 \AA)$. An intermediate length would be less physical considering the small vertical Debye-Waller factor and relatively large lateral coherence length of the diffracting domains. During least-squares refinement the most sensitive parameter was the tilt angle of the scattering cylinders. For the $21 \AA$ case, a very reasonable tilt angle uncertainty $\left(3 \pm 2^{\circ}\right)$ was found to fit the data consistent with previous findings from the lipid monolayers. Conversely, only a very narrow range of tilt angle $\left(4 \pm 0.2^{\circ}\right)$ fit the data in the $42 \AA$ case, an unrealistic constraint given the instrumental resolution as illustrated in Fig. 4. Together with the FWHM of the $\operatorname{rod}\left(L_{c} \approx 21 \AA\right)$ and better quality fit ( $\chi^{2}=1.85$ vs. 2.06), these findings suggest that the bilayer scatters as individual monolayers.

In earlier work on hydrated PC vesicles, Nagle and coworkers determined that the length of the scattering unit corresponded to two hydrocarbon chains, requiring each bilayer to scatter coherently [19]. Similarly, fluorescent studies of giant unilamellar vesicles of DPPE/DPPC mixtures show that solid phase domains span both the inner and outer leaflet of the vesicle membrane [20,21]. These studies demonstrate that free standing PE and PC bilayers form solid phase domains which are bilayer spanning. In contrast, our studies suggest that registry between the bilayer leaflets of DPPE is impeded by a solid support,

TABLE I. Tilt direction was fixed for bilayer models due to tilt angle close to $0^{\circ}$. This was done to minimize the number of fitting parameters to two for the bilayer models, the tilt angle and Debye-Waller factor ( $1.5 \AA$ for monolayers and $2 \AA$ for bilayers). NN $=$ tilt towards nearest neighbor.

\begin{tabular}{|c|c|c|c|c|c|c|c|c|c|}
\hline \multirow[b]{2}{*}{ DPPE } & \multirow[b]{2}{*}{$\begin{array}{c}\pi \\
(\mathrm{mN} / \mathrm{m})\end{array}$} & \multicolumn{4}{|c|}{ In-plane Bragg peaks } & \multicolumn{4}{|c|}{ Out-of-Plane Bragg rods } \\
\hline & & $\begin{array}{c}a, b(\AA) \\
\pm 0.002(\AA)\end{array}$ & $\begin{array}{l}\gamma \text { (deg.) } \\
\pm 0.2\left(^{\circ}\right)\end{array}$ & $\begin{array}{c}\text { Area per } \\
\text { molecule } \\
\pm 0.01\left(\AA^{2}\right)\end{array}$ & $\begin{array}{l}\text { Coherence } \\
\text { Length } L_{x y} \\
\pm 10.0(\AA)\end{array}$ & $\begin{array}{c}\text { Coherence } \\
\text { Length, } L_{c} \\
(\AA) \\
\text { (cylinder } \\
\text { height) }\end{array}$ & $\begin{array}{c}\text { Tilt } \\
\text { angle } \\
t\left(^{\circ}\right)\end{array}$ & $\begin{array}{l}\text { Tilt dir. } \\
\text { (from } \\
\text { NN) }\end{array}$ & $\begin{array}{c}\text { Red. } \\
\chi^{2}\end{array}$ \\
\hline \multirow[t]{3}{*}{$\begin{array}{l}\text { Mono } \\
\text { layers }\end{array}$} & 20 & $\begin{array}{l}4.883 \\
4.975\end{array}$ & $118.3^{\circ}$ & 42.79 & $\begin{aligned} L_{10} & =400 \\
L_{01} & =140 \\
L_{1-1} & =620\end{aligned}$ & $21 \pm 0.5$ & $21 \pm 2$ & $13.0^{\circ}$ & 7.0 \\
\hline & 30 & $\begin{array}{l}4.84 \pm 0.02 \\
4.90 \pm 0.02\end{array}$ & $\begin{array}{l}119.3^{\circ} \\
\pm 0.1\end{array}$ & $41.6 \pm 0.3$ & $\begin{aligned} L_{10,01} & =190^{\mathrm{a}} \\
L_{1-1} & =500\end{aligned}$ & $21 \pm 0.5$ & $15 \pm 2$ & 13.0 & 11.2 \\
\hline & 45 & $\begin{array}{l}4.809 \\
4.809\end{array}$ & $120.0^{\circ}$ & 40.06 & $L_{10.01,1-1}=570$ & $21 \pm 0.5$ & $4.5 \pm 2$ & $0^{\circ}$ & 18.8 \\
\hline Bilayer & $\begin{array}{l}\text { deposited } \\
\text { at } 45\end{array}$ & $4.84 \pm 0.01$ & $120.0^{\circ}$ & $40.57 \pm 0.17$ & $194 \pm 50$ & $\begin{array}{l}21 \text { fixed } \\
42 \text { fixed }\end{array}$ & $\begin{array}{l}3 \pm 2 \\
4 \pm 0.2\end{array}$ & $\begin{array}{l}0^{\circ} \text { fixed } \\
0^{\circ} \text { fixed }\end{array}$ & $\begin{array}{l}1.85 \\
2.06\end{array}$ \\
\hline
\end{tabular}

${ }^{\text {a}}$ Large uncertainty since Bragg peaks splitting is close to $q_{x y}$ resolution. Uncertainties are reported at the top of the column unless specified otherwise. 


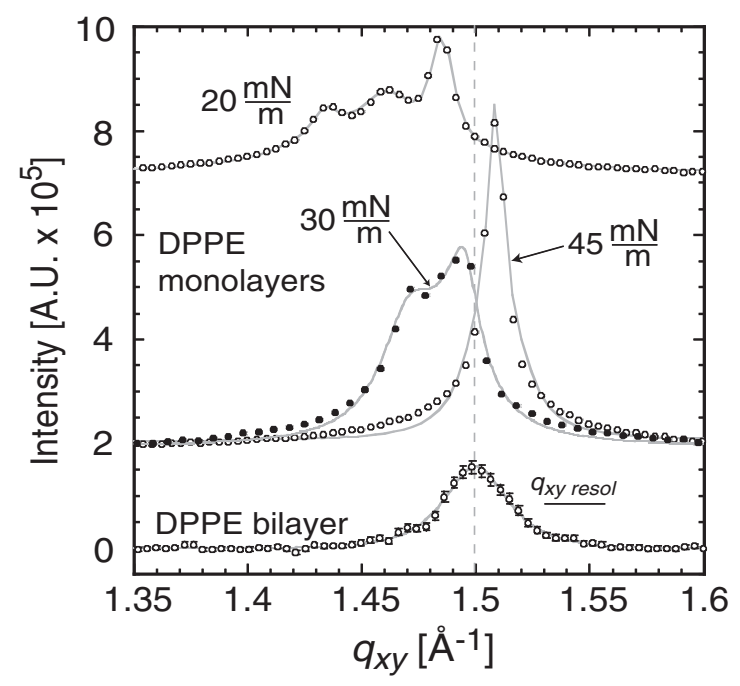

FIG. 3. Bragg peaks (integrated over $q_{z}$ ) from a DPPE bilayer membrane in comparison to the Bragg peaks from a DPPE monolayer at 20,30 and $45 \mathrm{mN} / \mathrm{m}$ at the air-water interface. Monolayers have been offset vertically for clarity. Analysis of the bilayer peaks reveal a hexagonal unit cell of $a_{H}=4.84 \pm$ $0.01 \AA$. Solid lines are fits to the Bragg peaks using Voight functions. The dashed line marks the center of the Bragg peak of the DPPE bilayer.

consistent with the difference in electron density (packing) of the two leaflets measured by reflectivity.

In conclusion, diffraction from a single lipid bilayer in bulk water has been observed for the first time and both inplane and out-of-plane structural information was obtained

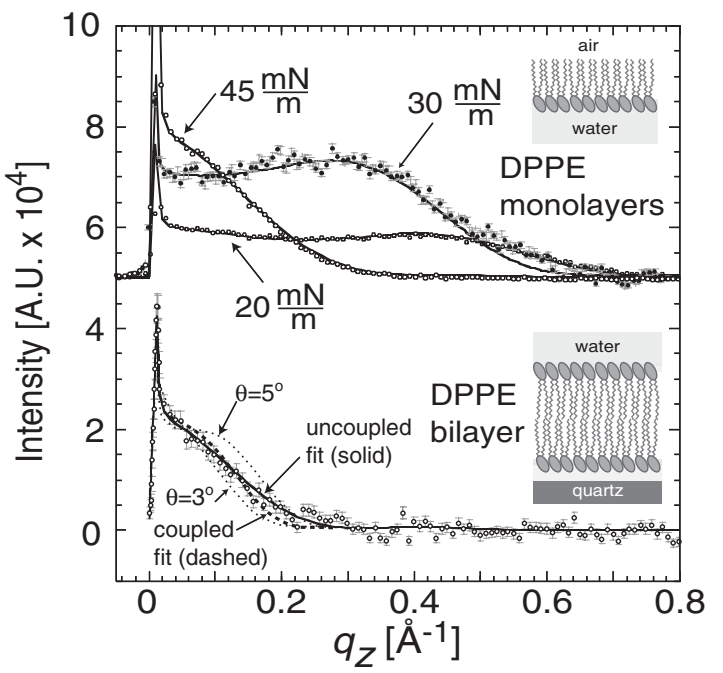

FIG. 4. Bragg rods (integrated over $q_{x y}$ ) from a DPPE bilayer in comparison to the Bragg rods from a DPPE monolayer at 20, 30 and $45 \mathrm{mN} / \mathrm{m}$ at the air-water interface. The sharp peak at $q_{z} \sim 0 \AA^{-1}$ is the Vineyard-Yoneda peak. Lines are fits to the models described in the text. Visual inspection of the DPPE bilayer rod reveals similar FWHM as in the monolayer case at $45 \mathrm{mN} / \mathrm{m}$. For the coupled model, small changes in the tilt angle $\left( \pm 1^{\circ}\right)$ dramatically impact the fit and the calculated Bragg rod FWHM (as shown by the light gray dotted curves). for a DPPE bilayer. Bragg rod analysis indicates that the two leaflets scatter independently. Further work on biomembranes will allow an assessment of solid phase domains in more complex systems. Since the exposure time for GIXD measurements is mere seconds, this methodology can be exploited to follow dynamic processes in real time.

This work was supported by Los Alamos National Laboratory under DOE contract No. W7405-ENG-36, the DOE Office of Basic Energy Science, and the National Science Foundation No. NSF-DMR \#0606564. C.E.M would like to thank LANL-LDRD and the Institute for Complex Adaptive Matter (ICAM). Work at APS is supported in part by the Office of Basic Energy Sciences of the U.S. Department of Energy and by the National Science Foundation Division of Materials Research. Use of the Advanced Photon Source is supported by the Office of Basic Energy Sciences of the U.S. Department of Energy under Contract No. W-31-109-Eng-38. We thank Dr. Kristian Kjaer for help with the monolayer scattering experiments and Dr. Doug Robinson for assistance with the bilayer measurements.

[1] K. Simons and W. L. C. Vaz, Ann. Rev. Bioph Biom 33, 269 (2004).

[2] P.F. Devaux and R. Morris, Traffic Engineering 5, 241 (2004).

[3] L. Chenghao, D. Constantin, and T. Salditt, J. Phys. Condens. Matter 16, S2439 (2004).

[4] A. Spaar, C. Munster, and T. Salditt, Biophys. J. 87, 396 (2004).

[5] C. E. Miller et al., Phys. Rev. Lett. 94, 238104 (2005).

[6] E. Novakova, K. Giewekemeyer, and T. Salditt, Phys. Rev. E 74, 051911 (2006).

[7] C. Reich et al., Rev. Sci. Instrum. 76, 095103 (2005).

[8] J. Daillant et al., Proc. Natl. Acad. Sci. U.S.A. 102, 11639 (2005).

[9] D. Y. Takamoto et al., Science 293, 1292 (2001).

[10] A. van der Lee, F. Salah, and B. Harzallah, J. Appl. Crystallogr. 40, 820 (2007).

[11] K. Kjaer, Physica (Amsterdam) 198B, 100 (1994).

[12] C. E. Miller et al., Z. Kristallogr. 220, 987 (2005).

[13] A. Guinier, X-ray Diffraction in Crystals, Imperfect Crystals, and Amorphous Bodies (W.H. Freeman, San Francisco, 1963).

[14] K. Kjaer et al., Thin Solid Films 159, 17 (1988).

[15] G. H. Wu et al., Biophys. J. 89, 3159 (2005).

[16] J. Marra and J. N. Israelachvili, Biochemistry 24, 4608 (1985).

[17] K. Y. C. Lee et al., Langmuir 14, 2567 (1998).

[18] T. R. Jensen and K. Kjaer, in Studies in Interface Science, edited by D. Möbius and R. Miller (Elsevier, Amsterdam, 2001), p. 205.

[19] S. Tristramnagle et al., Biophys. J. 64, 1097 (1993).

[20] L. A. Bagatolli and E. Gratton, Biophys. J. 78, 290 (2000).

[21] S. L. Veatch and S. L. Keller, Phys. Rev. Lett. 89, 268101 (2002).

[22] G. H. Vineyard, Phys. Rev. B 26, 4146 (1982). 